\title{
COMPARAÇÃO DE MÉTODOS PARA DETECÇÃO DE FOSFATASE ALCALINA E PEROXIDASE EM LEITE
}

\section{Comparison of methods for alkaline phosphatase and peroxidase detection in milk}

\author{
Felipe Nael Seixas ${ }^{1 *}$, Rafael Fagnani ${ }^{2}$, Edson Antônio Rios ${ }^{l}$, Juliana Ramos Pereira ${ }^{l}$, \\ Ronaldo Tamanini ${ }^{l}$, Vanerli Beloti ${ }^{1}$
}

\section{RESUMO}

Esse trabalho avaliou o desempenho de tiras colorimétricas para detecção de fosfatase alcalina e peroxidase em leite, comparando-as com um kit de reagentes para fosfatase alcalina e com a metodologia oficial para peroxidase. Para a comparação dos testes de detecção de fosfatase alcalina foram feitos quatro tratamentos adicionandose diferentes porcentagens de leite cru $(1 \%, 2 \%, 5 \%$ e $10 \%)$ ao leite pasteurizado, além de dois tratamentos controle. Foram analisadas 38 amostras em triplicata para cada tratamento. Para comparar o desempenho dos testes para peroxidase 80 amostras de leite pasteurizado foram avaliadas simultaneamente pela metodologia oficial e pelas tiras colorimétricas. O desempenho dos métodos para fosfatase alcalina foram distintos nos tratamentos com $1 \%$ e $2 \%$ de leite cru. Todas as tiras apresentaram alteração de cor, enquanto o kit de reagentes acusou a presença da fosfatase em apenas $2,63 \%$ e 5,26\% dos casos, respectivamente para cada tratamento. As tiras colorimétricas para fosfatase alcalina são mais sensíveis para identificação de pequenas quantidades quando comparadas ao kit de reagente. Já o desempenho dos testes para peroxidase, não apresentou diferença. Assim, as tiras para a detecção de fosfatase alcalina ou peroxidase mostraram-se eficientes, podendo substituir os métodos tradicionais.

Palavras-chave: pasteurizado; colorimétricas; reagentes; kit; análises; enzimática.

1 Universidade Estadual de Londrina (UEL),Centro de Ciências Agrárias, Departamento de Medicina Veterinária Preventiva, Laboratório de Inspeção de Produtos de Origem Animal, Rodovia Celso Garcia Cid, PR 445 km 380, Campus Universitário, 86051-990, Londrina, PR, Brasil. E-mail: a2fns@cav. udesc.br

2 Universidade Norte do Paraná (UNOPAR), Programa de Pós-graduação em Ciência e Tecnologia de Leite e Derivados, Londrina, PR, Brasil.

* Autor para correspondência. 


\begin{abstract}
This study evaluated the performance of strips for colorimetric detection of alkaline phosphatase and peroxidase in milk, comparing them with a kit of reagents for alkaline phosphatase and the official methodology for peroxidase. The samples were analyzed at the Laboratory Inspection of Products of Animal Origin, State University of Londrina. For the comparison tests for the detection of alkaline phosphatase four treatments were made by adding different percentages of raw milk $(1 \%, 2 \%, 5 \%$ and $10 \%$ ) in the pasteurized milk, plus two control treatments. Thirty-eight samples triplicate for each treatment were analyzed. To compare the performance of tests for peroxidase 80 pasteurized milk samples were evaluated simultaneously by official methodology and by colorimetric strips. The performance of the alkaline phosphatase were different for the treatments with $1 \%$ and $2 \%$ of raw milk which had all the strips change color as the reagent kit showed the presence of phosphatase in just $2.63 \%$ and $5.26 \%$ the cases, respectively for each treatment. The colorimetric strips for alkaline phosphatase are more sensitive for the identification of small quantities compared to the reagent kit. The performance of tests for peroxidase showed no difference. The strips for the detection of peroxidase or alkaline phosphatase were effective and can replace traditional methods.
\end{abstract}

Keywords: pasteurized; colorimetric; reagents; kit; analysis; enzymatic.

\section{INTRODUÇÃO}

A fosfatase alcalina (FA) e a peroxidase são enzimas naturalmente encontradas no leite cru e que, após a pasteurização, servem de indicadores para assegurar que o processo foi realizado adequadamente. Após o tratamento térmico, a fosfatase deve ser inativada e a peroxidase permanecer ativa (BRASIL, 2011).

A verificação da atividade residual da fosfatase alcalina como indicador da eficiência da pasteurização é amplamente utilizada no país e no mundo. Porém, poucos estudos descrevem com detalhes a cinética de inativação dessa enzima e a quantidade mínima da fosfatase alcalina detectável nos testes comerciais para o seu diagnóstico. A fosfatase alcalina pode indicar se a pasteurização atingiu a temperatura suficiente para eliminar os patógenos do leite, ou se ocorreu contaminação do leite pasteurizado com leite cru dentro do pasteurizador (TRONCO, 2008)
No Brasil, o teste para detecção de fosfatase alcalina recomendado é baseado na reação de compostos fenólicos resultantes da degradação enzimática com um substrato específico (fenil fosfato dissódico), permitindo identificar a atividade enzimática por uma reação colorimétrica. Para a detecção da peroxidase, o teste recomendado baseia-se na reação na qual a peroxidase ao hidrolisar o peróxido de hidrogênio, libera oxigênio, o qual transformará o guaiacol da sua leucobase para a forma corada (BRASIL, 2006). Atualmente estão disponíveis comercialmente conjuntos de reagentes (kits), além de tiras colorimétricas para a detecção da fosfatase alcalina (FA) e peroxidase, sendo que as pesquisas sobre as suas sensibilidades são escassas.

Este trabalho teve como objetivo avaliar o desempenho de tiras colorimétricas para detecção de fosfatase e peroxidase em leite, comparando-as respectivamente, com um kit de reagentes para fosfatase alcalina e com a metodologia oficial para peroxidase. 


\section{MATERIAL E MÉTODOS}

Foram utilizadas amostras de leite pasteurizado integral de diferentes laticínios, destinadas ao consumo na região norte do Paraná. Todas as amostras analisadas apresentavam-se dentro do prazo de produção de 48 horas e foram encaminhadas acondicionadas em caixa de isopor com gelo industrial ao Laboratório de Inspeção de Produtos de Origem Animal (LIPOA) da Universidade Estadual de Londrina (UEL).

\section{Fosfatase Alcalina}

Para avaliar a atividade da fosfatase alcalina no leite utilizaram-se quatro tratamentos adicionando-se diferentes porcentagens de leite cru $(1 \%, 2 \%, 5 \%$ e $10 \%$ ) ao leite pasteurizado. Também foram feitos dois tratamentos controles, um para fosfatase positiva, utilizando apenas leite cru, e outro para fosfatase negativa, utilizando apenas leite pasteurizado, o que totalizou seis tratamentos. Para a análise qualitativa da fosfatase alcalina foi comparado o kit de reagentes para fosfatase (Bioclin - Quibasa Química Básica, Belo Horizonte - MG, Brasil) na qual a fosfatase alcalina hidrolisa o substrato de timolftaleína monofosfato, liberando timolftaleína e fosfato inorgânico, e após 10 minutos a atividade enzimática é medida colorimetricamente. Quando a reação enzimática é inativada, a timolftaleína adquire cor azul. As tiras colorimétricas para detecção de fosfatase alcalina (Laborclin - Produtos para laboratório, Pinhais - PR, Brasil) utilizase de um substrato de para-nitrofenil-fosfato e tampão aminometil-propanol que em contato com a fosfatase alcalina presente na amostra adquire coloração amarela. Os resultados das leituras das tiras foram classificados em três intensidades a partir do tom amarelo: forte, média, fraca e sem alteração (branca, seguiu-se a recomendação do fabricante de incubar em estufa a $37^{\circ} \mathrm{C}$ e repetir a leitura).
Foram feitas repetições em triplicata para cada tratamento, com 38 diferentes amostras de leites pasteurizadas nas quais foram simuladas adição de leite cru conforme já mencionado.

Paralelamente, também foi feita a quantificação da fosfatase a partir do kit de reagentes em todos os tratamentos. Para isso, a solução que indica o resultado colorimétrico foi centrifugada a $5.000 \mathrm{rpm}$ por 10 minutos, com posterior filtração em membrana de celulose $(0,22 \mu \mathrm{m})$. A quantificação foi então realizada em espectrofotômetro semiautomático para bioquímica (Bioplus 2000) calibrado com o próprio padrão (40 UI de fosfatase por $\mathrm{mL}$ ) e branco do kit Bioclin. As leituras foram feitas em triplicatas, com 12 análises para cada um dos seis tratamentos, totalizando 72 leituras.

\section{Peroxidase}

Para verificar a atividade da peroxidase avaliou-se o desempenho de dois testes, as tiras colorimétricas (Laborclin) e a prova recomendada pelo Ministério da Agricultura, Pecuária e Abastecimento MAPA (BRASIL, 2006). Os testes utilizando as tiras colorimétricas foram realizados conforme a metodologia do fabricante. A presença da enzima é indicada pela coloração marrom na área sensibilizada da tira, 10 segundos após sua imersão na amostra. Considerou-se como positivo a mudança de cor na tira, do marrom claro ao marrom escuro, e como negativo as tiras que permaneceram brancas.

Para análise conforme a recomendação do MAPA foram transferidos $10 \mathrm{~mL}$ de cada amostra para um tubo de ensaio de $20 \mathrm{~mL}$, em seguida os tubos foram levados ao banhomaria por 5 minutos a $45^{\circ} \mathrm{C}$. Adicionou-se 2 $\mathrm{mL}$ da solução de guaiacol e 3 gotas de água oxigenada. O resultado positivo é dado pela formação de um halo de coloração salmão, em até 5 minutos. Foram consideradas como positivas as provas com formação de halos 
de coloração salmão claro a salmão escuro, e negativas, as provas sem formação de halo (brancas) (BRASIL, 2006).

No total, foram analisadas em duplicada 80 amostras de leite pasteurizado obtidas no comércio.

A proporção de resultados positivos e negativos para a detecção de fosfatase e peroxidase foi analisada pelo teste de Quiquadrado. Os valores das absorbâncias não tiveram distribuição normal no teste de Lilliefors e foram normalizados por conversão em $\log ^{10}$. Assim foram submetidos à análise de variância contemplando o efeito das diferentes porcentagens de leite cru adicionado ao pasteurizado sobre os valores da absorbância. Fatores com $\mathrm{p}<0,05$ foram posteriormente analisados pelo teste de Tukey a $0,5 \%$. Todas as análises foram realizadas pelo programa Statistica, versão 7.0 (STATSOFT., 2004)

Ambas as análises para fosfatase alcalina e peroxidase foram avaliadas imediatamente após o acréscimo das diferentes porcentagens de leite cru utilizado em cada tratamento.

\section{RESULTADOS E DISCUSSÃO}

\section{Fosfatase Alcalina}

$\mathrm{Na}$ interpretação dos resultados visuais das provas para avaliação da presença de fosfatase alcalina ativa no leite observou- se que os desempenhos dos métodos foram distintos para os tratamentos adicionados de $1 \%$ e $2 \%$ de leite cru (Tabela 1 ). Nesses tratamentos todas as tiras apresentaram alguma alteração de cor, indicando a atividade da enzima fosfatase alcalina. Entretanto, o kit de reagentes acusou a presença da fosfatase alcalina ativa em apenas $(2,63 \%)$ e $(5,26 \%)$ dos casos, nos tratamentos com $1 \%$ e $2 \%$ de leite cru, respectivamente. $\mathrm{O}$ kit de reagentes foi capaz de detectar visivelmente a presença da fosfatase ativa, apenas a partir da adição de $5 \%$ de leite cru (Tabela 1 ).

O desempenho dos testes foi semelhante nos tratamentos controle, com apenas leite cru ou com apenas leite pasteurizado, e também nos tratamentos com $5 \%$ e $10 \%$ de leite cru. Nesses tratamentos a proporção de resultados positivos e negativos não diferiu entre os métodos de detecção.

Quanto à intensidade de coloração obtida com as tiras os resultados podem ser visualizados na Figura 1. No tratamento contendo apenas leite pasteurizado as tiras não apresentaram nenhuma alteração de cor (brancas). Para os tratamentos com $1 \%$ e $2 \%$ de leite cru, a maioria das tiras mostrou coloração amarelo fraca e média em até uma hora. No leite cru e nos tratamentos com 5\% e $10 \%$ de leite cru, a maioria das tiras mostrou coloração amarelo forte imediatamente.

Tabela 1 - Presença de fosfatase alcalina em leite pasteurizado adicionado de diferentes porcentagens de leite cru, detectada pelo método de tiras e por kit de reagentes

\begin{tabular}{lcccccc}
\hline & Leite past. & $1 \%$ Cru & $2 \%$ Cru & $5 \%$ Cru & $10 \%$ Cru & Leite Cru \\
\hline $\operatorname{Tira}_{1}(+/-)$ & $(+/-)$ & $(+/-)$ & $(+/-)$ & $(+/-)$ & $(+/-)$ & $(+/-)$ \\
& $0 / 38^{\mathrm{a}}$ & $38 / 0^{\mathrm{a}}$ & $38 / 0^{\mathrm{a}}$ & $38 / 0^{\mathrm{a}}$ & $38 / 0^{\mathrm{a}}$ & $38 / 0^{\mathrm{a}}$ \\
Kit de reagentes $_{2}(+/-)$ & $(+/-)$ & $(+/-)$ & $(+/-)$ & $(+/-)$ & $(+/-)$ & $(+/-)$ \\
& $0 / 38^{\mathrm{a}}$ & $1 / 37^{\mathrm{b}}$ & $2 / 36^{\mathrm{b}}$ & $38 / 0^{\mathrm{a}}$ & $38 / 0^{\mathrm{a}}$ & $38 / 0^{\mathrm{a}}$ \\
\hline
\end{tabular}

Proporções seguidas de letras distintas na mesma coluna diferiram no teste $\mathrm{X}^{2}(\mathrm{p}<0,05)$

(+/-) presença/ausência da detecção da fosfatase alcalina

1. Tiras para fosfatase alcalina no leite Laborclin - Produtos para Laboratórios Ltda

2. Kit de reagentes para fosfatase alcalina Bioclin - Quibasa Química Básica Ltda 
O fabricante recomenda que a coloração amarela fraca das tiras seja considerada como resultado negativo para fosfatase alcalina. Os resultados sugerem que a interpretação do teste deva ser reavaliada, pois é importante considerar a primeira alteração na sua coloração. Isso aumenta sua sensibilidade e permite a detecção de menores quantidades da fosfatase alcalina.

O kit de reagentes (Bioclin) para fosfatase alcalina indica leitura dos resultados em até uma hora. No entanto, essa metodologia é menos prática e necessita de instrumentos e equipamentos como pipetas e banho-maria. A interpretação também é colorimétrica, com coloração azul para a presença de fosfatase alcalina (positivo) e amarela para a sua ausência (negativo). Tanto as leituras visuais do kit como a mensuração da absorbância mostraram que a sensibilidade do kit é menor do que a necessária para detectar as pequenas quantidades de fosfatase alcalina presentes no leite pasteurizado, ou seja, quando o mesmo possa se adicionado de pequenas quantidades de leite cru.

Comparando os resultados obtidos com as tiras e o kit de reagente, quando a coloração amarela fraca da tira foi considerada como resultado positivo, observou-se maior capacidade das tiras em detectar $(\mathrm{p}<0,05)$ resultados positivos nos tratamentos com $1 \%$ e $2 \%$ de leite cru adicionado ao leite pasteurizado. Portanto maior sensibilidade, em relação ao kit de reagentes que não alterou a cor observada a olho nu nestes tratamentos. A adição de $1 \%$ e $2 \%$ de leite cru ao leite pasteurizado também não foi detectada quando o kit de reagentes foi analisado pela espectrofotometria.

A média dos valores da absorbância entre o leite pasteurizado e os tratamentos adicionados de $1 \%$ e $2 \%$ não diferiram entre si (Tabela 2). A média de absorbância do tratamento adicionado de $10 \%$ de leite cru não diferiu do tratamento com $5 \%$, porém, foi diferente dos demais.

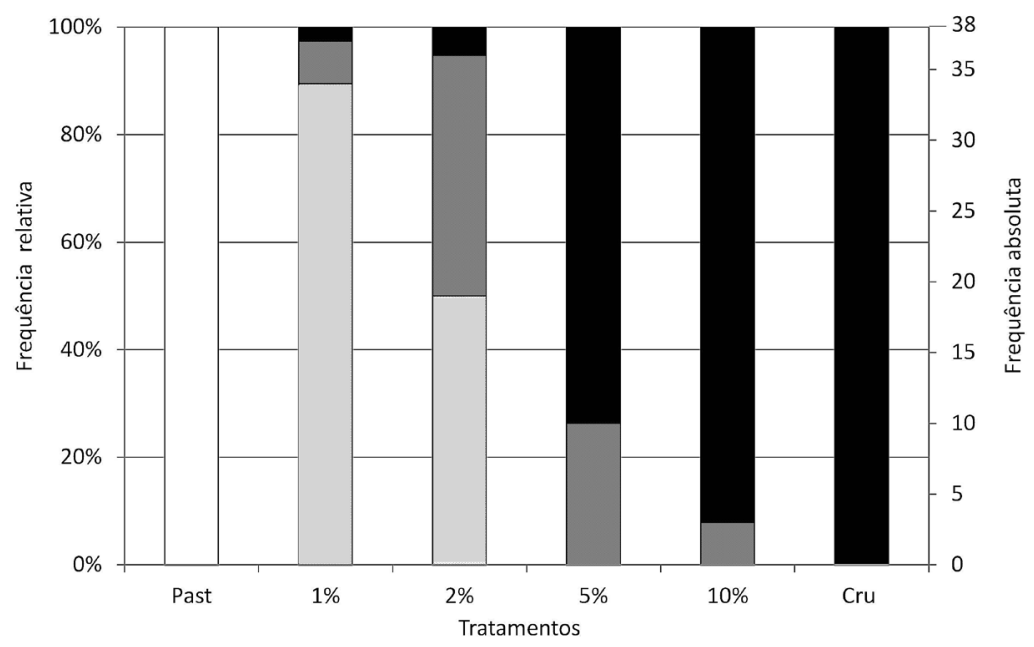

Intensidade da alteração de cor: $\quad$ Forte $\quad \square$ Média $\quad \square$ Fraca $\square$ Sem alteração

Figura 1 - Distribuição da coloração mostrada no teste em tiras (Laborclin) para detecção de fosfatase alcalina em leite, em seis diferentes tratamentos: leite cru, leite pasteurizado e leite pasteurizado adicionado com $1 \%, 2 \%, 5 \%$ e $10 \%$ de leite cru, em 38 repetições para cada tratamento 
Tabela 2 - Média (AU), média do logarítimo (Média Log Absorbância), desvio e erro padrão de valores de absorbância observados na solução colorimétrica do kit de reagentes para fosfatase alcalina em leite cru, leite pasteurizado e leite pasteurizado adicionado com diferentes porcentagens de leite cru

\begin{tabular}{lrccc}
\hline Tratamento $(\mathrm{n})$ & Média (AU) & $\begin{array}{c}\text { Média Log } \\
\text { Absorbância }\end{array}$ & Desvio padrão & Erro padrão \\
\hline Leite cru (12) & 308,33 & $2,48^{\mathrm{a}}$ & 0,09 & 0,02 \\
Leite pasteurizado (12) & 9,05 & $1,02^{\mathrm{d}}$ & 0,05 & 0,15 \\
$1 \%(12)$ & 9,68 & $1,27^{\mathrm{bd}}$ & 0,61 & 0,18 \\
$2 \%(12)$ & 13,19 & $1,27^{\mathrm{bd}}$ & 0,41 & 0,12 \\
$5 \%(12)$ & 32,24 & $1,59^{\mathrm{bc}}$ & 0,34 & 0,10 \\
$10 \%(12)$ & 46,39 & $1,75^{\mathrm{c}}$ & 0,38 & 0,11 \\
Total (72) & 77,50 & 1,56 & 0,63 & 0,07 \\
\hline
\end{tabular}

Médias seguidas de letras iguais não diferiram estatisticamente $(\mathrm{p}>0,05)$

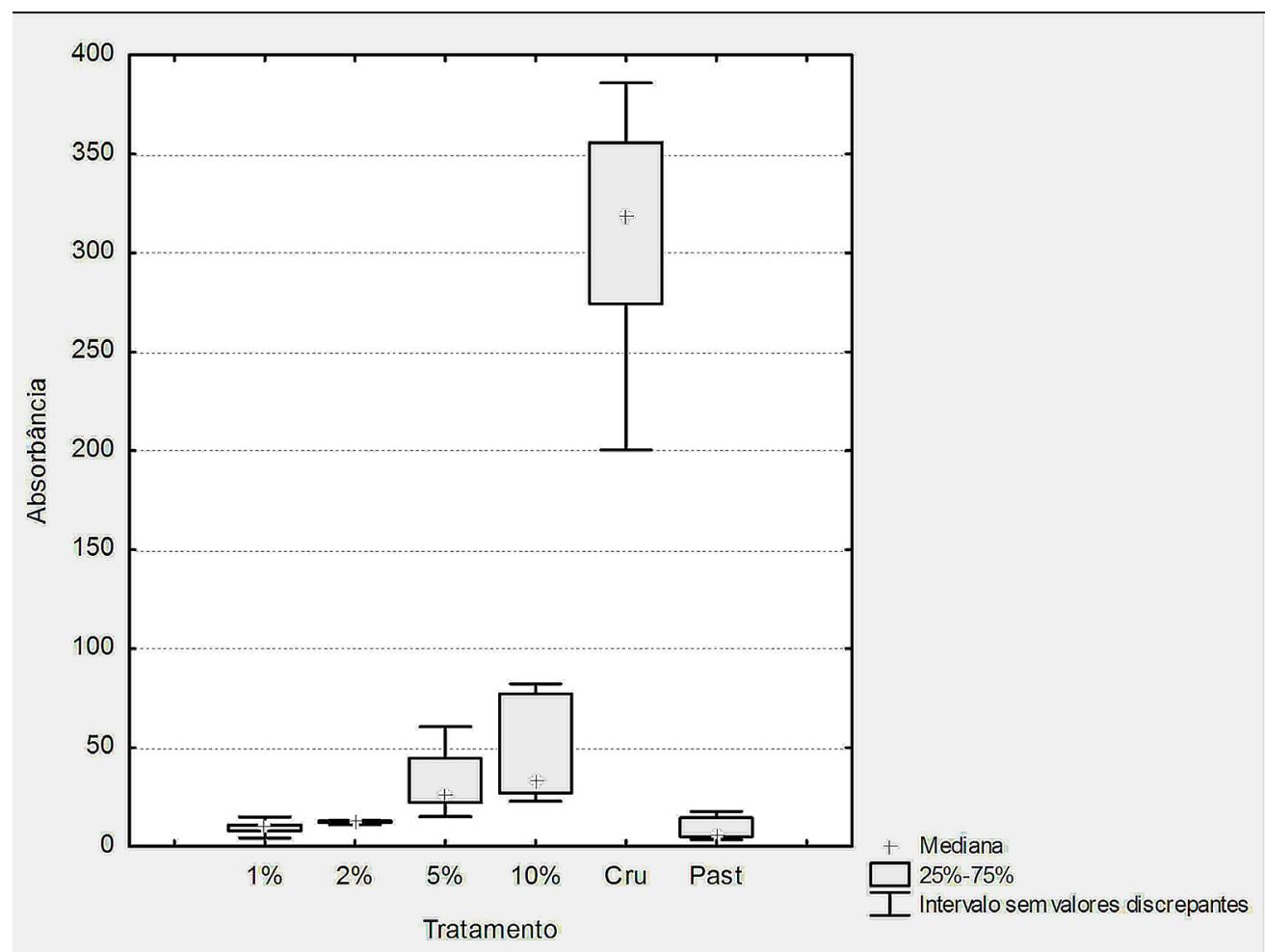

Figura 2 - Valores de absorbância (AU) para diferentes quantidades de leite cru adicionado ao pasteurizado 
O leite cru apresentou a maior variação para os valores de absorbância (Figura 2), com $95 \%$ dos valores observados entre 272 AU e 343AU, indicando que os níveis de fosfatase naturalmente presentes no leite variam consideravelmente. Segundo Murthy et al. (1992) a atividade da fosfatase pode variar conforme a sazonalidade, raça dos animais, período de lactação e volume de leite produzido.

Ainda pode ser observado que o desvio padrão aumentou conforme a quantidade de leite cru adicionada aos tratamentos, o que pode ter sido responsável pela falta de diferença estatística entre as médias de absorbância do leite pasteurizado e dos tratamentos com $1 \%$ e $2 \%$ de leite cru. Pode-se inferir que o kit de reagentes não é tão sensível quanto às tiras, mesmo quando a leitura dos resultados é observada em um espectrofotômetro.

A variação do binômio tempo-temperatura na pasteurização pode ter sido responsável por diferentes quantidades residuais de fosfatase alcalina ativa. Consequentemente pelo maior desvio e erro padrão dos valores de absorbância do leite pasteurizado quando comparados com os tratamentos $1 \%$ e $2 \%$, uma vez que esses leites foram obtidos do comércio e, portanto oriundos de diferentes laticínios. Painter; Bradley (1997) concluíram que oscilações no tempo-temperatura de pasteurização influenciam até $7 \%$ da atividade residual da fosfatase alcalina.

Considerando-se a quantidade máxima permitida de aeróbios mesofilos no leite cru pela Instrução Normativa 62 (BRASIL, 2011)


leite cru no leite pasteurizado pode representar a inclusão de até $12 \times 10^{5}$ bactérias $/ \mathrm{mL}$. Esses valores se tornam expressivos considerando que vários patógenos possam estar presentes, como Staphylococcus aureus, Campylobacter jejuni, Salmonella spp, Yersinia enterocolitica, Listeria monocytogenes e Escherichia coli (BELOTI et al., 1999; JAYARÃO; HENNING, 2001; NERO et al., 2008; TEBALDI et al., 2008; MATTOS et al., 2010).

\section{Peroxidase}

Observou-se que das 80 amostras analisadas de acordo com a técnica recomendada pela legislação brasileira (BRASIL, 2006), 75 (93,75\%) foram positivas e 5 $(6,25 \%)$ amostras de leites foram negativas. Ao se analisar as amostras com o uso das tiras comerciais (Laborclin) 68 (85\%) amostras de leite apresentaram-se positivas com a mudança de cor nas tiras do branco para tonalidades do marrom, e 12 (15\%) amostras analisadas foram negativas não apresentando mudança na cor da área sensibilizada da tira. Essa diferença de resultados entre os testes não foi significativa ( $p>0,05)$ segundo o teste de qui-quadrado. Ambas as análises realizadas são qualitativas, porém, de acordo com Behmer (1999) ao trabalhar com a pesquisa de peroxidase, na qual se usa a prova do guaiacol, observa-se a formação de uma coloração salmão. Apesar de não ser uma prova quantitativa, pode-se considerar que tonalidades fracas significam menor quantidade de peroxidase no leite.

O desempenho dos testes não apresentou diferença. As tiras comerciais podem ser usadas como prova de rotina, principalmente pela facilidade, rapidez e praticidade do teste em relação ao oficial, que necessita de instrumentos e equipamentos, como pipetas e banho-maria para sua realização. No entanto, devido a possível ocorrência de falsa negativos nos resultados das tiras, é recomendável que o teste oficial seja empregado como confirmatório nos casos em que as tiras comerciais apresentarem-se negativas.

Ausência de peroxidase em leites pasteurizados pode ser associada a fraudes por superaquecimento do leite para mascarar baixa qualidade microbiológica da matéria prima. Mas, também, pode ser decorrente de falhas durante o processamento do leite e no tempo/temperatura da pasteurização determinados na legislação brasileira. De qualquer forma, esse aumento do binômio tempo/temperatura poderá causar alteração 
no valor nutritivo, físico e químico deste alimento, com modificação e redução de suas qualidades sensoriais e nutricionais.

\section{CONCLUSÕES}

As tiras reagentes são mais sensíveis para identificação de pequenas quantidades de fosfatase alcalina no leite, se consideramos os resultados amarelo claro como positivos. O kit de reagentes pode não conseguir detectar a presença dessa enzima quando o leite pasteurizado estiver contaminado com quantidades de até $2 \%$ de leite cru.

O desempenho das tiras para detecção de peroxidase foi semelhante ao teste oficial preconizado pelo Ministério da Agricultura, Pecuária e Abastecimento. No entanto, por segurança, recomenda-se a confirmação dos resultados negativos das tiras pelo método convencional.

As tiras para a detecção de fosfatase alcalina e peroxidase mostraram-se eficientes, podendo ser usadas como alternativas às provas de rotina para detecção destas enzimas em leites pasteurizados destinados ao consumo à população humana.

\section{REFERÊNCIAS}

BELOTI, V. et al. Avaliação da qualidade do leite cru comercializado em Cornélio Procópio, Paraná. Controle do consumo e da comercialização. Semina: Ciências Agrárias, v.20, n.1, p.12-15, 1999.

BEHMER, M. L. A. Tecnologia do leite: queijo, manteiga, caseína, iogurte, sorvetes e instalações: produção, industrialização, análise. 13. ed. São Paulo: Nobel, 1999. 320 p.

BRASIL. Ministério da Agricultura, Pecuária e Abastecimento. Instrução Normativa no ${ }^{\circ} 68$, de 12 de dezembro de 2006. Oficializa os métodos analíticos oficiais físico-químicos, para controle de leite e produtos lácteos. Diário Oficial da República Federativa do Brasil, Brasília, 14 dez. 2006. Seção 1, p.8.
BRASIL. Ministério da Agricultura, Pecuária e Abastecimento. Instrução Normativa $n^{\circ} 62$, de 29 de dezembro de 2011. Aprova o Regulamento Técnico de Produção, Identidade e Qualidade do Leite tipo A, o Regulamento Técnico de Identidade e Qualidade de Leite Cru Refrigerado, o Regulamento Técnico de Identidade e Qualidade de Leite Pasteurizado e o Regulamento Técnico da Coleta de Leite Cru Refrigerado e seu Transporte a Granel, em conformidade com os Anexos desta Instrução Normativa. Diário Oficial da República Federativa do Brasil, Brasília, 30 dez. 2011. Seção 1, p. 6.

JAYARAO, B. M.; HENNING, D. R. Prevalence of foodborne pathogens in bulk tank milk. Journal of Dairy Science, v. 84, n. 10, p. 2157-2162, 2001.

MATTOS, M. R. et al. Qualidade do leite cru produzido na região do agreste de Pernambuco, Brasil. Semina: Ciências Agrárias, v.31, n.1, p.173-182, 2010.

MURTHY, G. K. et al. Alkaline phosphatase methods.In: R. T. MARSHALL. Standard methods for examination of dairy products. Washington, DC: American Public Health Association, 1992. p.413-431.

NERO, L. A. et al. Listeria monocytogenes and Salmonella spp. in raw milk produced in Brazil: occurrence and interference of indigenous microbiota in their isolation and development. Zoonoses and Public Health, v. 55, n.6, p.299-305. 2008.

PAINTER, C; BRADLEY, R. Residual alkaline phosphatase in milk subjected to various timetemperature treatments. Journal of Food Protection, v. 60, n.5, p. 252-530. 1997.

STATSOFT, Inc. STATISTICA (data analysis software system). version 7, 2004.

TEBALDI, V. M. R. et al. Isolamento de coliformes, estafilococos e enterococos de leite cru provenientes de tanques de refrigeração por expansão comunitários: identificação, ação lipolítica e proteolítica. Ciência e Tecnologia de Alimentos, v. 28 , n. 3, p. $753-760,2008$.

TRONCO, V.M. Manual para inspeção da qualidade do leite. 3 ed. Santa Maria: UFSM, 2008. 260p. 